\title{
DERIVAS CURRICULARES DE LAS POLÍTICAS DE CALIDAD EDUCATIVA EN LA UNIVERSIDAD COLOMBIANA
}

Mireya Uscátegui Caicedo Universidad de Nariño Colombia 
Mireya Uscátegui Caicedo

JEL: A20, A29

\section{Derivas curriculares de las políticas de calidad educativa en la universidad colombiana}

\section{Resumen}

Las políticas públicas de aseguramiento de la calidad educativa surgidas de las políticas transnacionales de globalización activaron desde la pasada década del noventa en Colombia, como en buena parte de la región, diversos procesos de renovación de las instituciones universitarias y de sus programas académicos cuyos currículos debieron adecuarse a los preceptos oficiales dando lugar a una suerte de derivas que hoy por hoy se constituyen en tema ineludible para comprender el particular desarrollo del campo curricular en estas naciones, tal y como lo han asumido ya autores en México, Argentina y en nuestro propio país. En esta perspectiva el presente artículo pone en consideración algunos elementos producto del análisis realizado sobre la manera cómo unas universidades colombianas han hecho observables las directrices nacionales en los programas de Derecho y Diseño Gráfico. La investigación en referencia de índole descriptivo-analítica, se abordó desde la teoría socio-crítica del currículo con un expreso interés hermenéutico y se adelantó con base en fuentes primarias de carácter normativo y en fuentes documentales de orden institucional de 24 universidades tanto públicas como privadas del país. La contrastación entre las fuentes hizo posible establecer que en el marco de la autonomía universitaria existen hoy distintos continentes curriculares producto de derivas bien con afirmaciones o bien con desplazamientos de la norma en variados sentidos, así como es posible también prever tensiones, fracturas y superposiciones en este campo.

Palabras clave: Currículum universitario; políticas públicas; imperativos curriculares; derivas curriculares.

\section{Dérives curriculaires des politiques de qualité de l'éducation à l'université colombienne}

\section{Résumé}

Les politiques publiques visant à garantir la qualité de l'éducation découlant des politiques de mondialisation transnationale sont actives depuis les années 90 en Colombie et dans sa région. Différents processus de rénovation des institutions universitaires et de leurs programmes académiques dont les programmes ont dû être adaptés aux réglementations officielles, conduisant à une sorte de dérive. Aujourd'hui, ils sont un sujet incontournable pour comprendre le développement particulier du domaine curriculaire dans ces pays, comme l'ont déjà supposé les auteurs du Mexique, de l'Argentine et de notre propre pays. Dans cette perspective, cet article examine certains éléments résultant de l'analyse effectuée sur la manière dont les universités colombiennes ont rendu les directives nationales observables dans les programmes de droit et de graphisme. La recherche en référence à une nature descriptive-analytique, a été abordée à partir de la théorie sociocritique du curriculum avec un intérêt herméneutique explicite et a été menée sur la base de sources primaires de nature normative et de sources documentaires d'ordre institutionnel de 24 publics et privés universités du pays. Le contraste entre les sources a permis d'établir que dans le cadre de l'autonomie universitaire aujourd'hui, il existe différents continents curriculaires du fait de dérives soit avec des affirmations soit avec des déplacements de la norme dans divers sens, ainsi qu'il est également possible de prévoir tensions, fractures et chevauchements dans ce domaine.

Mots clés: Programme universitaire; politique publique; impératifs curriculaires; dérives curriculaires. 
Mireya Uscátegui Caicedo

JEL: A20, A29

\title{
Curricular drifts of educational quality policies in the Colombian university
}

\begin{abstract}
Public policies for the assurance of educational quality arising from transnational globalization policies have been active since the 1990s in Colombia and its region. Various processes for the renewal of university institutions and their academic programs whose curricula had to be adapted to official regulations, leading to a kind of drift. Today they are an unavoidable subject to understand the particular development of the curricular field in these nations, as authors in Mexico, Argentina and in our own country have already assumed. In this perspective, this article considers some elements resulting from the analysis carried out on the way in which Colombian universities have made national guidelines observable in Law and Graphic Design programs. The research in reference to a descriptive-analytical nature, was approached from the socio-critical theory of the curriculum with an express hermeneutic interest and was carried out based on primary sources of a normative nature and documentary sources of institutional order from 24 public and private universities from the country. The contrast between the sources made it possible to establish that within the framework of university autonomy today there are different curricular continents as a result of drifts either with affirmations or with displacements of the norm in various senses, as well as it is also possible to foresee tensions, fractures and overlaps in this field.
\end{abstract}

Keywords: University program; public policy; curriculum imperatives; curriculum drifts. 


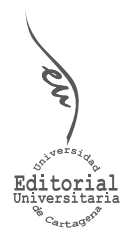

Artículo de Reflexión

\section{Derivas curriculares de las políticas de calidad educativa en la universidad colombiana'}

INFORMACIÓN DEL ARTíCULO

Recepción del artículo: 03/04/2018

Concepto de evaluación: 17/06/2018

Aceptación del artículo: 23/07/2018
Mireya Uscátegui Caicedo²

Universidad de Nariño, Colombia

\section{INTRODUCCIÓN}

El campo del currículum universitario en Colombia, como en varios países de la región, revela un inusitado desarrollo a partir de la década del noventa del siglo pasado por cuenta de las políticas públicas orientadas al aseguramiento de la calidad educativa, corriente que nacida de las políticas transnacionales del seno de la globalización con sus nutrientes de modernidad y neoliberalismo, dejó a su paso una afluencia de reformas estructurales (Coraggio, 1995; Lora, 2001; Chomsky, 2001; Arocena, 2004; Nieto y Díaz Villa, 2005; Rodrigo, 2006; De Alba, 2009; Tarabini y Bonal, 2011) dentro de las cuales la educación ocupa un lugar prioritario ${ }^{3}$.

Para la implementación de estas políticas y de sus correspondientes normas y en desarrollo de la Constitución Nacional de 1991 se creó en 1992 el Consejo Nacional de Acreditación -CNA- organismo central encargado de hacerlas operativas a través de lineamientos que articulados en factores e indicadores encarnan las características de calidad educativa y obran en la práctica como verdaderos imperativos curriculares, tanto por su origen exógeno como por su naturaleza normativa, exponiendo a las universidades a la perentoria tarea de adecuar los currículos a los estándares establecidos en ellas.

\footnotetext{
1. Artículo producto del proyecto de investigación: "El currículo, tendencias y metodologías de construcción en programas universitarios de Colombia" realizado con el apoyo de la Universidad de Nariño.

2. Doctora en Ciencias de la Educación. Universidad de Nariño. Profesora Titular de la Facultad de Artes. Universidad de Nariño. Líder del Grupo de investigación. "Currículo y Universidad". Universidad de Nariño. Contacto: mireyadesign20@udenar.edu.co

3. Orozco S., L.E. (2012) señala que en la década del noventa se emprendieron 18 reformas en trece países de América Latina.
} 
Dicha tarea tiene lugar en un contexto en el que pueden considerarse al menos tres aspectos a partir de los cuales es hipotéticamente dable que en el proceso de adopción de la norma hayan tenido lugar una suerte de derivas curriculares de distinta especie y que resultan por demás significativos para la comprensión del desarrollo del campo. El primero de estos aspectos radica en el hecho de que para entonces la universidad colombiana estaba más cerca de los ideales de la modernidad que de los de la modernización que servía de marco a las mencionadas políticas, amén de que el curricular había sido un tema tradicionalmente ausente de las grandes discusiones académicas en el ámbito universitario colombiano, razón por la cual para la mayoría de las facultades y programas académicos los terrenos de este campo y sus lenguajes resultaban poco conocidos y menos explorados.

El segundo aspecto a considerar es la declaración por primera vez en el país, de la autonomía universitaria como derecho constitucional consagrado así en la nueva Carta Política de 1991, suceso que al interior de las instituciones constituía el resultado de históricas luchas por lo que la implementación del sistema de acreditación provocó variadas opiniones como aquellas que rechazaron sus lineamientos por considerarlos atentatorios de la autonomía universitaria; el tercer aspecto nos remite al panorama de una educación universitaria que mediante el discurso gubernamental y desde el sector productivo se juzga por carecer de pertinencia como dimensión de calidad del servicio educativo (Orozco Silva, 2010), lo que a la sazón se constituyó en factor de presión social para las instituciones haciéndose ineludibles las reformas curriculares en busca de la ponderada acreditación de calidad, lo que presuntamente favorecería la incorporación acrítica del bagaje lingüístico de los estándares de calidad y el riesgo de la improvisación en el tema por el uso apresurado y poco minucioso de términos que como flexibilidad y pertinencia (Orozco Fuentes, 2009) entre otros, prefiguran los currículos en el marco de la preconizada calidad educativa.

Cabe advertir cómo la misma noción de calidad que inspira a estas políticas genera no pocos debates debido al carácter polisémico, complejo e ideologizado del término (Aguerrondo, 1993; Orozco Silva, 2010; Roncal, 2014; Sarzuri-Lima, 2014; Vega Cantor, 2014) cuyo sentido lato pudo ser en general compartido en el ámbito universitario pero no así su significado expreso dado el trasfondo político que compromete a la reforma educativa con un determinado modelo de desarrollo y un proyecto particular de sociedad reafirmando así mismo el talante imperativo (Castro-Gómez, 2007b) de los factores de calidad curricular.

En esta perspectiva el Grupo de investigación "Currículo y universidad" de la Universidad de Nariño ha abordado desde el año 2000 el análisis de los fenómenos suscitados como efecto de la implementación de dichas políticas bajo el entendimiento del currículum como campo relacional conflictivo (Bourdieu, 1990) en cuya configuración se desarrollan variados procesos (Stenhouse, 1991) en los que intervienen disímiles intereses que hacen presumible la presencia de tensiones entre lo prescrito desde las agencias normativas y lo deseable por parte de los agentes curriculares institucionales (Martínez Delgado, 2009).

Para el caso presente se registran determinados aspectos relativos a algunas derivas curriculares evidenciadas a través de una de las recientes investigaciones adelantada en el Grupo ${ }^{4}$ con el propósito de comprender el desarrollo del campo curricular en Colombia a partir del análisis de las tendencias conceptuales de mayor incidencia en la normatividad

4. La investigación en referencia "El currículo, tendencias y metodologías de construcción en programas universitarios de Colombia" fue realizada por Isabel Goyes Moreno y Mireya Uscátegui de J. con el auspicio de la Universidad de Nariño (Pasto-Colombia) 
nacional y establecer la manera cómo algunas universidades han hecho observables tales directrices en 24 programas universitarios de Derecho y Diseño Gráfico y/o Visual pertenecientes a universidades públicas y privadas, antiguas y nuevas, de la capital y de provincia, en orden al cumplimiento de los lineamientos de calidad definidos desde 1995 por el Consejo Nacional de Acreditación, año que marca el primer extremo temporal del estudio que abarcó un período de veinte años.

La conveniencia de esta investigación radica en el reconocimiento de que nuestro país enfrentado a grandes exigencias de calidad y pertinencia educativa, se encuentra en trance de consensos sobre el significado y alcance de nociones curriculares de nuevo cuño en el terreno universitario y que presuntamente pondrían en juego el ejercicio de la autonomía educativa toda vez que los variados sentidos de calidad marcan a su vez diferencias de sentido en conceptos generalmente consagrados en los proyectos institucionales como equidad, pertinencia, progreso, y desarrollo (Castro-Gómez, 2007a), aspectos que si bien están implícitos en estudios generales sobre las políticas públicas de mejoramiento de la calidad en la educación superior tales como los que se han ocupado de los procesos de desarrollo de la norma así como de los y resultados e implicaciones de su aplicación en varios países de la región dentro de los que se cuentan Argentina, Bolivia, Brasil, Colombia, Chile, México y Perú, entre otros, expresamente en Colombia pocas veces el currículum universitario como campo asociado a dichas políticas ha sido materia específica de estos estudios.

En virtud de la naturaleza social del problema planteado, el objeto se auscultó desde el paradigma cualitativo de la investigación y se abordó desde la teoría socio-crítica del currículo (Kemmis, 1993; Stenhouse, 1991; De Alba, 1991,1998) en razón a que ella permite comprender el currículo como un entramado en el que se definen implícitamente las relaciones que el Estado entabla con la sociedad a través del sistema educativo y bajo el conjetura de que no obstante que el currículo constituye el instrumento por excelencia para la implementación de las políticas públicas, las instituciones universitarias en virtud de su autonomía pueden apelar a sus sentidos de educación, a su teleología y a su propia experiencia que como capital habrá de traducirse en los currículos.

El estudio tuvo un carácter descriptivo-analítico con un manifiesto interés hermenéutico recurriendo para el efecto a dos tipos de fuentes documentales primarias: a) las de índole normativa de orden nacional representadas por todas aquellas que desde la Constitución Nacional de 1991 incluida ésta, prefiguran el contexto retórico de las regulaciones y lineamientos oficiales para el aseguramiento de la calidad en la educación superior en Colombia y, b) las curriculares de orden institucional universitario tales como los proyectos educativos institucionales y los de programas académicos de Derecho y Diseño Gráfico/Visual ${ }^{5}$, y a fuentes secundarias como el estudio diagnóstico hecho por el CARAD (2008) sobre los programas de Diseño en Colombia a la luz de la Resolución 3463 de 2003 que regula el funcionamiento de estos programas en el marco de las políticas de aseguramiento de la calidad. 


\section{CONSTRUCCIÓN TEÓRICO-CONCEPTUAL DEL OBJETO}

La afirmación del currículum como campo en el sentido bourdiano del término incita a develar tanto la complejidad de las relaciones internas que lo configuran como los intereses que prefiguran, para el caso presente el campo curricular universitario, en tanto objeto al que subyacen relatos de poder harto diversos provenientes de esferas no siempre coincidentes como las de orden político-transnacional, normativo-nacional, regulativo-institucional y epistemológico-disciplinar.

En consecuencia el abordaje del objeto en tal carácter de complejidad precisa de marcos argumentativos que desde presupuestos como los de la teoría socio-crítica desborden las tradicionales concepciones técnico-instrumentales y las meramente formales del currículum para dar paso a aquellas que permitan el análisis de los fenómenos que tienen ocurrencia en los procesos de traducción entre una y otra esfera discursiva.

Según los presupuestos bourdianos (Bourdieu, 1990) el campo curricular puede definirse como un universo intermedio sujeto a tensiones surgidas por las relaciones de agencias -transnacionales y gubernamentales-, y agentes -instituciones, docentes y estudiantesque provistos de sus respectivos haberes participan en la producción, selección, reproducción y difusión de las ciencias convirtiendo el currículum en un campo relacional conflictivo razón por la cual su análisis implica el discernimiento crítico de los contextos y los procesos que lo configuran, desde los generativos histórico-políticos hasta los de su desarrollo procesual-práctico (De Alba, 1998).

Así lo estima Díaz Villa (2007) al distinguir en principio dos tensiones básicas ocurridas como parte de la dinámica de los procesos de reforma curricular universitaria: una externa generada por las relaciones entre las instituciones educativas y el sector productivo, y otra interna generada por las relaciones entre los propios agentes académicos.

Estas dos tensiones hacen parte, en cierta medida, de la situación que hoy se vive en muchas instituciones de educación superior de América Latina las cuales, por lo general, deben responder a las demandas permanentes de adaptación y cambio propuesto desde un campo ampliamente determinante en la educación superior como es el campo internacional donde se juegan intereses económicos, políticos, ideológicos o científico-tecnológicos que pueden considerarse, directa o indirectamente, determinantes en los procesos de una reforma.

[...] El currículo es un ejemplo de la tensión entre diferentes fuerzas que actúan directa o indirectamente en su producción: la autoridad del Estado, las fuerzas del mercado y la oligarquía académica. (Clark, 1983)

[...] Hemos dicho en otro lugar que el campo de la educación superior está constituido básicamente por agentes, agencias, discursos y prácticas que ocupan diferentes posiciones en la estructura del campo y entran en diferentes relaciones externas e internas. (Díaz Villa, 2007, p. 60, 61, 68)

Por su parte en el marco de la teoría socio-crítica formulada por Kemmis (1993) el currículum es una construcción socio-histórica compleja que deviene en un conjunto de interacciones dialécticas entre la teoría prescriptiva o pre-activa y la práctica activa, relaciones que operan mediante procesos igualmente complejos de negociación y decisión a través de los cuales se hacen manifiestos los discursos simbólicos de relaciones de poder y los códigos que mediatizan la realidad socio-educativa. Tratándose de una construcción social su arquitectura prevé la intervención de diversos agentes educativos con sus 
complejas variables personales, lo que hace del currículum un ámbito problemático, un ininteligible campo de estudio socio-político, cultural y educativo. Bajo los mismos postulados Stenhouse (1991) proclama el carácter proyectual, perfectible e hipotético, por ende consecuente y razonablemente flexible y contextualizado del currículum.

El currículum es en tal modo una "[...] síntesis de elementos culturales [...] que conforman una propuesta político educativa, pensada e impulsada por diversos grupos y sectores sociales, cuyos intereses son diversos y contradictorios" (De Alba, 1991, p. 67). A partir de allí agencias y agentes serán reconocibles como sujetos sociales del currículum entendidos como grupos que sostienen determinados proyectos sociales y determinan el currículum mediante mecanismos explícitos o implícitos de lucha, negociaciones o imposiciones.

Se comprende así la construcción del currículum como un proceso de determinación socio-política que entraña la misma complejidad de la sociedad; en tal contexto los sujetos sociales son distinguibles según intervengan en uno de los tres ámbitos de su configuración (De Alba, 1991): a) el de los sujetos sociales de determinación curricular en el que se deciden los aspectos estructurantes del currículum en un nivel macro de concreción y orientación; es el espacio de intervención del Estado, las agencias económicas multinacionales, los gremios profesionales y los sectores productivos, entre otros; b) el ámbito de los sujetos sociales de la estructuración formal del currículo en el que se formaliza, estructura, planifica y concreta el currículum en su nivel meso y en observancia con lo dispuesto por el Estado y por las demás agencias; es la esfera de actuación de los organismos institucionales y disciplinares tales como consejos universitarios, academias, comités o equipos de autoevaluación, planeación y diseño curricular; c) el ámbito de los sujetos sociales de desarrollo curricular, el de los cuerpos docentes y estudiantiles que concretan el nivel micro del currículum a través del cual activan y ejecutan lo dispuesto por los dos sujetos sociales precedentes.

El Estado en tanto sujeto social determina el currículum a través de políticas públicas educacionales que entrañan un sistema de decisiones conexas y de cursos de acción que los gobiernos adoptan a fin de garantizar la educación deseable en un contexto sociohistórico específico, lo que incluye la definición de fines y medios para la solución de situaciones conflictivas propias de las dinámicas sociales o según el caso presente para la implantación de medidas y reformas estructurales que acondicionen los cambios socio-económicos que un determinado modelo de desarrollo requiere. Desde lo presupuestado por las teorías socio-crítica y de la estructuración (Giddens, 1995) se revela así mismo cómo en su condición de política pública tales decisiones en lo que respecta a su conceptualización y a sus alcances están sistemáticamente vinculadas a acciones emplazadas al logro de metas tendientes a mantener una relación estratégica y reproductora entre el Estado y la sociedad a través de la educación.

Las políticas educacionales suponen en particular al menos tres elementos (Espinosa, 2009) que para el análisis que nos ocupa fungen como dispositivos discursivos: el argumento que la justifica, el propósito que la anima, y los supuestos teóricos que la explican para hacerla operativa. La fuente de esos propósitos que se presumen colegidos de los fines de la educación, puede por el contrario residir en una órbita de intereses externos, verbigracia en una teoría económica o social, en una filiación política o religiosa, o en una tendencia cultural.

A su vez varios autores de afiliación socio-crítica y otros funcionalistas como los citados por Espinosa (2009), distinguen tres niveles en el desarrollo de estas políticas: el de la 
retórica, enunciada en discursos oficiales de alcance nacional o internacional contentivos de las metas educacionales previstas; el de la política impulsada a través de leyes de educación o de decretos más específicos y operativos, y el de la política efectivamente implementada mediante acciones puntuales como evidencia de los cambios prescritos. Traducido este desarrollo al campo del currículum puede explicarse cómo en el tránsito semántico de un nivel a otro, las políticas educativas se convierten en discursos prescriptivos cuya fuerza normativa hace que su bagaje conceptual opere como acervo de imperativos curriculares.

Tanto el Estado como las instituciones educativas en tanto sujetos sociales instauran de este modo unas dimensiones generales que "[...] dan cuenta de los aspectos esenciales del currículum, al tiempo que señalan sus límites [y] conforman una parte constitutiva importante del mismo" (De Alba, 1998, p.57). Pueden distinguirse entonces, entre otras, una dimensión social amplia de alcance cultural, político, social, económico e ideológico, y una dimensión institucional en la que se define su carácter como entidad universitaria y su identidad propiamente dicha. Son estas dos dimensiones las que auscultadas permiten reconocer los imperativos curriculares y presumir los movimientos, desplazamientos y re-conceptualizaciones, obrados en las dinámicas de su implementación.

Para la teoría socio-crítica la dimensión social-política se funda en el reconocimiento del currículum como proyecto político que define y orienta la práctica educativa desplazándolo de la presunta asepsia de las teorías empírico-analíticas, mientras la dimensión social-económica determina al currículum desde los intereses del sector productivo de donde exporta su discurso técnico y estratégico estableciendo relaciones de dependencia y colonización entre el aparato productivo, el mercado de trabajo y la universidad. Por su parte la dimensión institucional representa el espacio por antonomasia del currículum universitario, "[...] en el que se concreta la síntesis de elementos culturales (conocimientos, valores, creencias, hábitos y actitudes) que conforman una determinada propuesta académico-política" (De Alba, 1998, p.64).

En el juego de las relaciones entre las dimensiones descritas surgen hipotéticamente múltiples desplazamientos discursivos y consecuentes re-conceptualizaciones: de lineamientos normativos generales a fundamentaciones institucionales, de los contextos retóricos a los contextos de la práctica curricular y de ésta a estrategias singulares, en un engranaje cuya dinámica depende de cuánto se acerquen o cuánto se alejen los sentidos adoptados durante la evolución de la norma. De esta manera se advierte cómo los movimientos internos del campo curricular estriban en el capital semántico específico (Bourdieu, 1986) que cada agente apuesta, trátese del peso conceptual del capital económico de las agencias mundiales y del capital político del Estado, trátese del capital social de las instituciones o del capital cultural de los docentes (Alzamora, Beltrán y Aguilar, 2013); son justamente esos movimientos semánticos los que concitan la noción de derivas curriculares ${ }^{6}$ para entender los cambios acontecidos en los meso currículos respecto al macro currículo como efecto de la implementación de las normas que certifican la calidad de un currículo universitario.

\footnotetext{
6. Se adopta aquí el concepto de derivas siguiendo el eje nocional que nos remite al fenómeno de las derivas continentales, proceso cuyos accidentes (plegamientos, cabalgamientos, fracturas, apilamientos...) podrían fungir por su analogía, como categorías de análisis para las reconfiguraciones curriculares originadas como efecto de la aplicación de las políticas y los lineamientos de calidad en la educación superior universitaria.
} 
Nos referimos en consecuencia a los variados tipos de desplazamientos ocurridos al incorporar los cambios normativos desde el terreno oficial -un continente de conceptos propios de las políticas económicas trasnacionales relativos a la calidad-, al terreno de lo curricular institucional-un continentedeacervosy tradicioneseducativas habitualmente distantes de esas nociones- razón por la cual las universidades deben afrontar el reto de la adaptación de la norma bien sea mediante la re-conceptualización crítica, mediante su apropiación acrítica o en una mixtura ecléctica entre una y otra, casos todos cuyos resultados podrán mostrar un mapa de lo sucedido en el meso currículo y que se pretenden reconocer como derivas curriculares observables a través de desplazamientos de sentido con alejamientos o con fusiones de uno y otro continente, con plegamientos totales a la norma o con fracturas de ella, con apilamientos de fragmentos de uno y otro continente sin que alguno se afecte, con cabalgamientos -equivalentes a cambios superficiales, vale decir retóricos, por la compresión de la norma- o con erosiones teórico conceptuales para dar paso a nuevas configuraciones.

Uno de los propósitos que alienta el análisis del currículum desde las teorías críticas, principalmente de las que se fundamentan en la Nueva Sociología de la Educación, es la elucidación de estos desplazamientos discursivos por cuanto según la orientación semántica y epistemológica de esta corriente el lenguaje es una construcción social que promueve determinados intereses, valores concretos e ideologías específicas. De conformidad con esta corriente crítica los discursos que giran en torno a la educación y sus correlatos curriculares estarían delimitados por los supuestos teóricos y por las relaciones tanto socio-políticas como socio-económicas de los sujetos sociales que los produce (Penalva, J., 2008). Por lo tanto, en el contexto de la globalización en el que se generan las políticas públicas de aseguramiento de la calidad educativa para los países de la región se comprenden los supuestos teóricos y teleológicos que orientan hoy la educación universitaria y se advierte la procedencia ideológica de los imperativos curriculares.

Un factor común en el desarrollo de estas políticas ha sido la incorporación de nuevos lenguajes alusivos a la calidad educativa, concepto que al ser parte del discurso oficial resulta estrechamente vinculado al alcance de metas y a la evidencia de estándares internacionales traducidos en características curriculares de calidad, tales como las condiciones de flexibilidad, interdisciplinariedad, pertinencia, formación por competencias e internacionalización. Sin embargo, desde la perspectiva crítica del currículum los atributos de flexibilidad y pertinencia social son condiciones connaturales al currículum, muy distantes de la noción de calidad mediada por la eficacia y la eficiencia como argumentos discursivos propios del modelo económico-político que inspira las políticas globales.

En este punto cabe señalar el notable incremento de la investigación curricular en América Latina particularmente en los últimos decenios, destacándose los índices de producción académica en países como México, Brasil, Chile y Argentina. Los trabajos de Ángel Díaz-Barriga en México, por ejemplo, abarcan un amplio espectro del campo, desde los aspectos teóricos e históricos hasta su desarrollo práctico, así como las propuestas de diseño y evaluación curricular.

A su vez los estudios de la también mexicana Alicia de Alba, abordan el análisis sociocrítico del currículum universitario en los actuales contextos históricos y de cara a la realidad latinoamericana aportando de este modo elementos teóricos y conceptuales para el entendimiento de los fenómenos socio-culturales y políticos que circundan o determinan el campo curricular en la región. 
La investigadora mexicana Frida Díaz Barriga (2005) distingue en su país cinco ejes temáticos en la producción curricular correspondiente a la década del noventa, dentro de los cuales conviene citar el de la conceptuación de la esfera de lo curricular. La misma autora revela también cómo dentro de las tendencias de innovación curricular se destacan los enfoques administrativos de planeación estratégica que se fundan en los indicadores de calidad tales que garanticen alcanzar la eficacia, eficiencia y excelencia académica, junto con las innovaciones que buscan incorporar al currículo características propias de la globalización educativa como flexibilidad, pertinencia, interdisciplinariedad, internacionalización y transversalidad, y las que acogen el enfoque por competencias.

Por su parte la autora mexicana Beatriz Orozco Flórez en un estudio publicado en al año 2009 da cuenta del origen de la noción de flexibilidad y de su llegada al campo curricular con lo que reconoce la importancia del contexto histórico en el que éste arribo se produce debido a las variadas connotaciones que proliferan al plantear términos que como el de flexibilidad, cabría decir que así también los de calidad y pertinencia, se caracterizan por su polisemia y revelan de paso la inestabilidad del lenguaje curricular, aún en estado de constitución.

Otra investigación que ausculta las reformas curriculares en la región latinoamericana es la realizada por Guillermo Ferrer (2004) quien hace el análisis de lo ocurrido durante los años noventa en cuatro países: Perú, Colombia, Chile y Argentina, con relación a los mecanismos para la elaboración, la implementación curricular, la evaluación de los resultados de aprendizaje y el uso de las evaluaciones para el mejoramiento de las políticas y las prácticas curriculares. El autor destaca los empeños nacionales para hacer de estas reformas un proceso participativo ${ }^{7}$, lo que constituye un ingrediente común a los cuatro países en los que con todo se encuentran también significativas diferencias.

En Colombia en general son escasos los estudios específicos acerca de la manera como se han incorporado los nuevos lenguajes a los currículos universitarios; de allí que toman importancia los avances teórico-conceptuales de autores nacionales como Mario DíazVilla en torno a la constitución del campo y la flexibilidad curricular, Sergio Tobón sobre competencias en el marco de la teoría de la complejidad, Alberto Malagón acerca de la pertinencia curricular y la internacionalización y el CARAD -Comité Académico de la Asociación Colombiana Red Académica del Diseño-

\section{DERIVACIONES DEL ESTUDIO}

Las políticas públicas para el aseguramiento de la calidad de la educación superior en Colombia le imprimieron en efecto una inusual dinámica al campo del currículum universitario produciendo en él movimientos que pueden percibirse en tres momentos: 1) el de la gestación de tales políticas en las que se determinan los cambios curriculares, 2) el de su formalización e implementación normativa, y 3) el de la incorporación de sus imperativos en el ámbito institucional de las universidades en donde se surten toda

7. Respecto al carácter participativo es preciso recordar aquí que la construcción de los currículos desde un enfoque socio-crítico supera las limitaciones conceptuales y metodológicas de las teorías técnicas para contribuir desde la ciencia al reconocimiento de dos aspectos primordiales: el de contextualizar históricamente la esencia social de cada proyecto curricular y, el de adelantar los procesos de manera cooperativa y auto reflexiva buscando la transformación de la realidad social. (Kemmis, 1993) 
especie de reformas, adecuaciones, adaptaciones y otras acciones curriculares puntuales necesarias para dar cuenta del cumplimiento de los cambios prescritos.

Así, a partir del contexto de gestación de estas políticas en virtud tanto de la condición exógena de algunos de los sujetos sociales como del modelo socio-económico desde el cual se pretenden, se producen movimientos en el terreno de la identidad universitaria a manera de una deriva curricular con desplazamientos en la autonomía que tienden a desdibujar la identidad universitaria en reciprocidad con su misión social, fenómeno comparable con lo ocurrido en otros países de la región según lo describe Mollis, M. (2003) en alusión al flujo de reformas en el marco de similares políticas durante los años noventa en Argentina, Bolivia, Brasil, Chile y México:

En la llamada década perdida para el desarrollo económico, para la equidad y para la justicia social, las universidades han perdido su razón d'être. Al quebrarse el contrato social con el Estado y ser arrojadas a las fuerzas del "todopoderoso mercado", las universidades públicas y privadas de América Latina comenzaron a transitar la "mercadotecnia" como una alternativa para recuperar su debilitado financiamiento y buscar una nueva identidad.

[...] La simplicidad del contexto internacional de las reformas contrastaba con la complejidad de la tarea a la que se abocaban los gobiernos de Argentina, Bolivia, Brasil, Chile y México, fundamentalmente. El sentido de la gran transformación universitaria en América Latina en los '90 fue el cambio de la identidad pública universitaria -necesaria durante el imperio del Estado Benefactor- hacia una identidad en tránsito en el contexto global del Estado empresarial. (p.9 - 10)

Por lo descrito estamos ante una subordinación del modelo de educación universitaria que, para Castro Gómez (2017a) es un rasgo de la postmodernidad y conduce a estas instituciones a una crisis de legitimidad en la medida en que ellas, ante su incapacidad de independizarse de los imperativos del mercado global, comienzan a acomodarse a ellos.

Desde la teoría socia-crítica esto constituiría una deriva curricular de fractura y plegamiento de la identidad universitaria teniendo en cuenta que como parte ella las instituciones universitarias están llamadas a cumplir una función en términos de la contextualización social conocimiento a partir de los referentes locales y las necesidades particulares de transformación, función que sería suplantada por la nueva identidad empresarial, contribuyendo de este modo a acentuar los procesos de colonización antes que los de emancipación.

En el segundo momento, concerniente a la formalización e implementación normativa, se advierten una particular expansión de la dimensión política del currículum que conforme a lo presentado por Mollis (2003), hace parte del catálogo de indicadores de las novedades acontecidas en Argentina, Brasil, Bolivia, Chile, México y Venezuela durante la década del noventa: el surgimiento de "Leyes de educación superior (marcos regulatorios) en escenarios institucionales con tradición autónoma" (p.10), y la sincrónica aparición de reformas preceptivas.

En Colombia el hecho se manifiesta en el movimiento normativo del campo durante los años 1991 y 2015 como pudo percibirse tras el análisis de lo acontecido en la regulación curricular general y en la específica para los programas de Derecho y Diseño Gráfico/ Visual cuya síntesis se presenta en la siguiente tabla. 
Tabla 1.

Marco normativo de la educación superior en Colombia (1991 - 2015)

NORMA

OBJETO DE LA NORMA

Constitución Política de 1991

Establece la Educación Superior como servicio público, de función social y garantiza la autonomía universitaria.

Ley 30 de 1992

Organiza el servicio público de la Educación Superior y crea el Sistema Nacional de Acreditación y el Consejo Nacional de Acreditación (CNA).

Reglamenta el proceso de acreditación voluntaria, define la acreditación como acto público de reconocimiento sobre la calidad de los programas y preescribe el proceso de acreditación.

Acuerdo CESU

06 de 1995

Se adoptan las políticas generales de acreditación.

Decreto 2566 de 2003

Establece las condiciones mínimas de calidad y demás requisitos para el ofrecimiento y desarrollo de programas académicos de educación superior.

Resolución MEN

2768 de 2003

Define las características específicas de calidad para los programas de pregrado en Derecho,

Resolución MEN

3463 de 2003

Define las características específicas de calidad para la oferta y desarrollo de los programas de formación profesional en Diseño.

Decreto 1295 de 2010

Reglamenta el registro calificado, la oferta y el desarrollo de programas académicos de educación superior.

Acuerdo 03 de 2011

Establece los lineamientos para la acreditación de programas de instituciones acreditadas institucionalmente.

Acuerdo 02 de 2012

Establece la apreciación de condiciones iniciales de acreditación de programas académicos.

Acuerdo 03 de 2014

Ordena los lineamientos para la acreditación institucional.

Circular MEN N 03 de 2015
Se expide información sobre el proceso de acreditación de alta calidad para pregrado.

Nota: Elaboración propia. 
El estudio indica unas derivas curriculares diferenciadas entre los programas de Derecho y los de Diseño Gráfico/Visual debido a que en el primer caso esta disciplina ha estado históricamente sometida a la regulación primero por parte de los poderes monacal y eclesiástico de España, y luego por parte del Estado colombiano como una constante de los tiempos y los espacios que controla la formación en el área, los planes de estudio, los contenidos y los textos de aprendizaje, los métodos de enseñanza y los sistemas evaluativos adoptados por los docentes.

Entre tanto para el caso del Diseño Gráfico/Visual, si bien sus antecedentes de la se remontan a los talleres de artes y oficios de la época colonial en la que su regulación se determinaba a través de Cédulas Reales y de Bulas papales, la profesionalización propiamente dicha comienza apenas en 1963 con la apertura del primer programa de esta modalidad a nivel superior en la Universidad Nacional de Colombia. Desde entonces ni la formación en la disciplina ni su ejercicio profesional habían sido regulados por el Estado, salvo en el marco de normas generales para la educación superior como el Decreto 3191 de 1980 que reglamenta las unidades de labor académica evaluables y las distribuye porcentualmente en tres campos curriculares: el social y humanístico, el de fundamentación científica e investigativa y el de formación específica. A partir de allí no se habían instaurado normas generales de educación universitaria, ni de alcance disciplinar hasta las que devienen de las políticas públicas para el aseguramiento de la calidad.

Ya en el año 2003 aparecen para las dos disciplinas las normas que regulan de una parte la formación en Derecho y de otra la formación profesional en Diseño. En cada una de ellas se consagran los perfiles profesionales, los componentes curriculares y las competencias de formación que irán a transformar el currículum universitario con la incorporación de los imperativos curriculares ya informados.

El ingreso del discurso regulador y a través de éste de los imperativos curriculares a un contexto universitario caracterizado por la autonomía y la autorregulación, ha generado movimientos que dado el carácter obligatorio de la norma, ocasionan en general derivas curriculares de desplazamiento relativo o cabalgamiento de los florecientes lenguajes con los que se verbaliza y formaliza la calidad en su sentido oficial, por sobre las habituales prácticas de regulación institucional y disciplinar sin que aquellos logren transformar en su totalidad el currículum universitario.

Es el tercer momento, el de la incorporación de los imperativos al ámbito universitario, en el que se verifica un mayor número de derivas curriculares habida cuenta del conjunto de movimientos semánticos y procesuales que la adopción de la norma produce.

Dentro de los procesuales, los más elocuentes son el del advenimiento de la cultura de la autoevaluación curricular y la planeación estratégica como oportunidad de mejoramiento y con fines de acreditación de calidad -una práctica no siempre generalizada a nivel universitario-, y el del carácter participativo con el que se aborda ahora la construcción de las propuestas curriculares, a diferencia de la tradicional rutina propia de la teoría técnica del currículo según la cual se replicaba la división del trabajo entre los expertos curriculares quienes elaboraban los currículos, y los docentes quienes aparentemente lo reproducían en la práctica.

Mollis (2003) refiere entre las transformaciones observables en los cinco países que fueron objeto de su estudio, las relacionadas con reformas académicas como el acortamiento de 
carreras en aras de la eficacia y la eficiencia del currículum universitario, el otorgamiento de títulos intermedios, la flexibilización curricular por la modalidad de créditos y "la importación de modelos basados en la adquisición de competencias profesionales" (p.10).

Por nuestra parte del análisis de las tendencias y las metodologías de construcción curricular se desprenden unos aspectos generales comunes y otros particulares. Entre los primeros tenemos que:

- El tema curricular permeó la formación universitaria.

- La elaboración del documento maestro que debe remitirse al Ministerio de Educación Nacional, tanto para la creación como para el funcionamiento de cualquier programa universitario, obliga a reflexionar con mayor o menor profundidad en la teoría curricular, en sus enfoques, en sus innovaciones y en su ejecución.

- La noción de competencias, se plantea desde una perspectiva resignificada tal como lo planteó el Proyecto Tuning América Latina que más allá del simple saber hacer en contexto, incorpora la formación para el ser y el saber de cada disciplina.

- Las características de flexibilidad, interdisciplinariedad y pertinencia, tanto por exigencia normativa como por los propios requerimientos del proceso formativo, se incluyen al estructurar los currículos. No obstante, la forma de materializarlos resulta en muchas ocasiones mecánica y repetitiva.

- Los avances iniciales en materia curricular producto de los mandatos legales han sufrido una especie de estancamiento en la medida en que los documentos maestros se convirtieron en escritos formales que no reflejan procesos creativos, reflexivos y comprometidos, sino que son meras transpolaciones de procesos vividos en otros contextos que se adoptan sin análisis crítico.

- Es innegable el impacto de las políticas de aseguramiento de la calidad en los procesos de fundamentación y estructuración del currículum universitario; de hecho, en aras del cumplimiento de las normas que desarrollan tales políticas, el tema curricular se ha puesto a la orden de los asuntos que ahora le son propios a la educación superior y que antes solo parecían pertenecer a las Facultades de Educación y a la educación básica y media.

- Los procesos de autoevaluación de los programas y las instituciones han modificado las prácticas de determinación y estructuración curricular en las universidades al presuponer ejercicios de índole participativa con la vinculación de docentes, estudiantes y egresados, así como de los actores pertenecientes al sector empresarial que a cada campo profesional le compete.

- El principio de flexibilidad curricular encontró graves obstáculos dado el esquema curricular tradicional que impuso una visión vertical del proceso de aprendizaje y un orden estricto en el nivel de conocimientos, razón que explica las dificultades de su implementación. 
- Con frecuencia muchos programas tratan de manera superficial conceptos como el de flexibilidad curricular reduciendo esta a la inclusión en el plan de estudios de un listado de asignaturas electivas; la interdisciplinariedad por su parte se limita a adoptar asignaturas o seminarios sobre temáticas complementarias a las propias de la disciplina; la pertinencia no pasa muchas veces de ser una breve referencia a las problemáticas del entorno.

- Se observan lugares comunes tanto en la definición de las políticas institucionales referidas a las nociones de flexibilidad, integralidad, pertinencia, interdisciplinariedad e internacionalización, como en las formas curriculares que se adoptan para su materialización, lo que podría sugerir el riesgo de estar ante una definitiva estandarización formal de los currículos con desconocimiento de los respectivos entornos culturales y ante una adopción más técnica que dialéctica de estos atributos curriculares.

- Uno de los problemas curriculares habituales al que no son ajenos los programas universitarios en Colombia, es como lo ha definido L. Stenhouse (1991), el hiato existente entre la retórica curricular y su encarnación en la práctica del aula. Los procesos participativos en la construcción y estructuración de los currículos suelen contribuir, de algún modo, a la ruptura de esta tendencia negativa.

En lo particular, los programas de Derecho analizados revelan las siguientes novedades:

- Los modelos socio crítico y de competencias son los más difundidos entre los programas de Derecho en Colombia.

- Los avances en materia curricular resultan estrechamente vinculados con las directrices trazadas desde el Ministerio de Educación las cuales enfatizan en la necesidad de implementar el aprendizaje en competencias, objetivo que se involucra en la fundamentación, la estructuración y la operativización curricular.

- Se encuentra que un $58 \%$ de los planes de estudio en Derecho son de naturaleza asignaturista mientras el $42 \%$ de los programas presenta otras formas de estructuración curricular en las que bajo las categorías de campo, contexto, núcleo, ciclo o componente, se agrupan materias, seminarios o módulos tendientes a formar de acuerdo con la identidad institucional, los que se desarrollan a lo largo de la carrera.

- En la totalidad de los programas está previsto el proceso formativo en investigación, diferenciándose en el número de seminarios o materias ofrecidas en cada uno de ellos.

- En más del 70\% de los documentos maestros puede observarse que la concreción del componente de flexibilidad curricular se encuentra en las asignaturas electivas u optativas las cuales generalmente son las que involucran el aspecto humanístico también denominado complementario.

Entre tanto respecto a los programas de Diseño Gráfico/Visual el proceso de incorporación de la norma ha generado reconfiguraciones curriculares las cuales revelan que: 
- Si bien la totalidad de estos programas incluyen las condiciones y características curriculares previstas en las correspondientes normas nacionales, tales como la formación de competencias básicas, no se evidencia una tendencia específica hacia un determinado modelo curricular.

- Se percibe la presencia de dos nuevos estructurantes curriculares para los programas de esta disciplina: las competencias y los componentes, los cuales podrían modificar las prácticas pedagógicas y evaluativas.

- Es apreciable la tendencia de los programas hacia la incorporación de valores propios de la disciplina y de su ejercicio profesional.

- En cuanto a la organización de contenidos curriculares se encuentran programas estructurados por organizadores amplios como áreas académicas o temáticas; ciclos básicos o componentes de fundamentación o de formación de alcance institucional o general, disciplinar, específica, profesional, o de profundización; componentes de integración, de inter o de transdisciplinariedad; núcleos, ciclos o ejes transversales como los de investigación; componentes de flexibilidad con electivas de tipo disciplinar o interdisciplinar. Estos organizadores que clasifican el conocimiento y lo secuencian según su relación con los perfiles, objetivos y procesos de enseñanza y de aprendizaje, se descomponen en materias, asignaturas, talleres, seminarios, actividades obligatorias y actividades optativas.

En el panorama expuesto se perfila entonces un enjambre de movimientos que suponen la ocurrencia de derivas curriculares:

a) de fractura y plegamiento como las ocurridas con la contracción del currículum como efecto de la presión ejercida por las políticas globales educativas de aseguramiento de la calidad educativa y de su carga semántica devenida de un modelo económico cuyo interés suplanta el deber ser de la institución universitaria;

b)de desplazamientos relativos entre el terreno de las políticas y el curricular sin que uno $u$ otro se afecten, como los que acontecen con el imperativo de flexibilidad curricular que si bien se inserta retóricamente en los distintos planos del currículum, lo hace de manera instrumental por medio de los denominados créditos optativos o mediante la adopción de estructuras en apariencia innovadoras a nivel universitario tales como las modulares pero coexistentes con las tradicionales y rígidas asignaturistas, con lo cual re-conforman el currículum solo en su aspecto observable sin erosionar el interés estratégico de la política trasnacional ni procurar la re-constitución epistemológica del currículum tal que desfigure los límites disciplinares para dar paso a estructuras abiertas a la inter y transdisciplinariedad.

c) de transformaciones procesuales mediante la asimilación de nuevas prácticas que se incorporan a la cultura universitaria como las de autoevaluación y las orientadas a la construcción colaborativa del currículum alterando rutinas y procesos estáticos sin perder por eso el carácter autónomo que define la identidad universitaria. 
d) de reconfiguración del campo curricular mediante la re-conceptualización crítica de términos que como el de pertinencia suele incorporarse al currículum pero con un sentido social contextualizado en el entorno que resulta políticamente opuesto a la pertinencia laboral que habita el territorio oficial; tal deriva aporta tanto a la re-configuración semántica como a la evolución del campo.

Es necesario aclarar que la caracterización presentada no tiene aspiraciones de ser absoluta ni suficiente, sino de aportar argumentos para la reflexión y el análisis de la realidad curricular colombiana en procura de nuevos y mejores panoramas de elucidación.

\section{A MODO DE CONCLUSIONES}

Según el análisis realizado se prefigura un mapa curricular producto del desarrollo de las políticas públicas de aseguramiento de la calidad que como quiera han dinamizado el campo convirtiéndose en una importante oportunidad para el surgimiento de epistemologías regionales así como para la construcción de nuevos sentidos derivados de la realidad latinoamericana, sin que esto signifique en sí mismo ni el mejoramiento per se de las condiciones o de las características de calidad educativa, ni la transformación cualitativa del currículum universitario, toda vez que aún deben analizarse los efectos reales de unas políticas cuyos correlatos de calidad no alcanzan a auscultarse más que en a través de indicadores de orden administrativo y formal.

No sobra advertir que los discursivos son solo un tipo de desplazamiento posible en el tránsito de la retórica normativa a la activación curricular en el aula; otras derivas deseables serían las de la estructura organizativo-académica de las universidades sin las cuales no será fácilmente posible la configuración de nuevos continentes curriculares como los inter y transdisciplinarios dadas las actuales condiciones de lo que Castro-Gómez (2007a) llama "arborización de la estructura universitaria" (p.84) cuando según el autor tal estructura funciona a su vez a partir de la "estructura arbórea del conocimiento" (p.81) refiriéndose respectivamente a la organización académico-administrativa de facultades y departamentos en los que se alojan los programas académicos y a la estructura disciplinaria de los epistemes que consagra y reproduce el modelo de la colonialidad del saber mediante la desagregación/jerarquización del conocimiento convertido en disciplinas, estructuras que operan como barreras infranqueables para acceder a la construcción de currículos diferentes a los monológicos y monodisciplinarios, tal y como lo precisa Castro Gómez (2007a):

El primer elemento común que me parece identificar es la estructura arbórea del conocimiento y de la universidad. Ambos modelos favorecen la idea de que los conocimientos tienen unas jerarquías, unas especialidades, unos límites que marcan la diferencia entre unos campos del saber y otros, unas fronteras epistémicas que no pueden ser transgredidas, unos cánones que definen sus procedimientos y sus funciones particulares.

[...] Lo que hace una disciplina es, básicamente, recortar un ámbito del conocimiento y trazar líneas fronterizas con respecto a otros ámbitos del conocimiento [...] ignorando sus conexiones con todas las demás.

[...] Pero la hybris del punto cero se refleja no sólo en la disciplinarización del conocimiento, sino también en la arborización de la estructura universitaria. La mayoría 
de las universidades funcionan por "facultades", que a su vez tienen "departamentos", que a su vez tienen "programas". Las facultades funcionan como una especie de hogares de refugio para las epistemes.

[...] Rara vez los profesores pueden moverse entre un departamento y otro, mucho menos entre una facultad y otra, porque son como prisioneros de una estructura universitaria, esencialmente fracturada. (p81, 83, 84)

Frente a lo expuesto no cabe duda de que así como hoy nos encontramos ante un panorama de positivos cambios curriculares promovidos desde las políticas nacionales, el camino por recorrer exige aplicarse epistemológica y académicamente en los ámbitos de su enseñanza y de su evolución cualitativa en contexto con las urgencias socio-culturales, científicas y político-económicas de la región.

\section{REFERENCIAS BIBLIOGRÁFICAS}

Aguerrondo, I. (1993). La calidad de la educación: Ejes para su definición y evaluación. OEA/ Revista Interamericana de Desarrollo Educativo. En: Revista La Educación [revista en Internet] [Acceso 03 de junio de 2010]; año XXXVII, número 116, III, 561-578. Bs. As., Argentina. Disponible en: http://campus-oei.org/calidad/aguerrondo.htm

Alzamora, G.; Beltrán, F.; Aguilar, l. (2013). La configuración del campo de la formación docente permanente en el nivel medio en la Argentina: estudio del caso de la provincia de La Pampa. Tesis Doctoral - Universidad de Valencia (Esp.). [Acceso 12 de abril 2015]. Disponible en http://roderic.uv.es/bitstream/handle/10550/32096/TESIS\%20 ALZAMORA.pdf?sequence=

Angulo, R. y Orozco, B. (2007). Alternativas metodológicas de intervención curricular en la educación superior. México: Plaza y Valdés S.A de C.V.

Arocena, R. (2004). Las reformas de la educación superior y los problemas del desarrollo en América Latina. Revista Educ. Soc., Campinas, 25 (8): (915-936). Disponible en: http://www.scielo. br/pdf/es/v25n88/a13v2588.pdf

Bourdieu, P. (1986). The forms of capital. Handbook of Theory and Research for the Sociology of Education. New York: Greenwood

Bourdieu, P. (1990). Sociología y cultura. México: Editorial Grijalbo, S.A.

CARAD, Comité Académico de la Asociación Colombiana Red Académica de Diseño-RAD-, Uscátegui, M. y otros. (2008). Fundamentos del Diseño en la universidad colombiana. Fase1. Medellín: Universidad Pontificia Bolivariana -UPB-

Castro-Gómez, S. (2007a). Decolonizar la universidad. La hybris del punto cero y el diálogo de saberes. Disponible en: http://www.ram-wan.net/restrepo/decolonial/14-castrodescolonizar\%20la\%20universidad.pdf

Castro-Gómez, S. (2007b). Michel Foucault y la colonialidad del poder. Revista Tabula rasa No.6: 153-172, enero-junio 2007. Disponible en: http://www.revistatabularasa.org/ numero-6/castro.pdf 
Coraggio, J. L. (1995). Las propuestas del Banco Mundial para la educación: ¿sentido oculto o problemas de concepción? Ponencia presentada en el Seminario "O Banco Mundial e as Politicas de Educação no Brasil”, organizado por Açao Educativa, Sao Paulo, 28-30 junio, 1995. Recuperado de: coraggioeconomia.org/jlc/archivos\%20para\%20descargar/ SANPABLO.pdf

Chomsky, N. (2001). La globalización en América Latina. Trabajo presentado en la Cátedra Latinoamericana Julio Cortázar, Guadalajara, Jal., México.

De Alba, A. (1998). Curriculum: crisis, mito y perspectiva. Buenos Aires: Miño y Dávila SRL

De Alba, A. (2009). El currículum universitario en la crisis estructural generalizada. En: Orozco

Fuentes, B. (coord.) (2009). Currículum: experiencias y configuraciones conceptuales en México. México: IISUE-UNAM / Plaza y Valdés

Díaz Villa, M. (2007). Reforma Curricular: Elementos para el estudio de sus tensiones. En: Angulo

Ferrer, G. (2004). Las reformas curriculares de Perú, Colombia, Chile y Argentina: ¿Quién responde por los resultados? Lima: GRADE. (Documento de trabajo 45). Disponible en: https:// ageconsearch.umn.edu/bitstream/37735/2/ddt45.pdf

Flórez López, J. y Flórez Gómez, G. (2016). La política pública de educación en Colombia y Chile: gestión del personal docente y reformas educativas globales. Revista Criterio Jurídico Garantista; 9(15), 68-85. Disponible en: revistas.fuac.edu.co/index.php/criteriojuridico garantista/article/view/643/596

Giddens, A. (1995), La constitución de la sociedad. Bases para la teoría de la estructuración. Buenos Aires: Amorrortu

Kemmis, S. (1993). El currículum: Más allá de la teoría de la reproducción. Madrid: Morata

Lora, E. (2001). Las reformas estructurales en América Latina: qué se ha reformado y cómo medirlo. Banco Interamericano de desarrollo. Disponible en: https://publications.iadb.org/es/ publicacion/15696/las-reformas-estructurales-en-america-latina-que-se-ha reformado-y-como-medirlo

López Segrera, F. (2003). El impacto de la globalización y de las políticas educativas en los sistemas de educación superior de América Latina y el Caribe. En: Mollis, M. (comp.) (2003). Las universidades en América Latina: ¿reformadas o alteradas? La cosmética del poder financiero. Buenos Aires (Argentina): Clacso, Asdi. 39-58

Martínez Delgado, M. (2009). Sujetos sociales y poderes de decisión en el currículum universitario. En: Orozco Fuentes, B. (coord.) (2009). Currículum: experiencias y configuraciones conceptuales en México. México: IISUE-UNAM / Plaza y Valdés

Ministerio de Educación Nacional de la República de Colombia. (2006). Lineamientos para la Acreditación de Programas. Colombia: CNA

Ministerio de Educación Nacional de la República de Colombia (2016). Compendio estadístico de la educación superior colombiana. Bogotá, Imprenta Nacional de Colombia. Disponible en: https://www.mineducacion.gov.co/1759/articles-360739_recurso.pdf

Ministerio de Educación Nacional de la República de Colombia, Sistema Nacional de Información de la Educación Superior SNIES, Módulo Consultas. Disponible en: https://snies. mineducacion.gov.co/consultasnies/programa 
Mollis, M. (comp.) (2003). Las universidades en América Latina: ¿reformadas o alteradas? La cosmética del poder financiero. Buenos Aires (Argentina): Clacso, Asdi. 9-16

Nieto, L. M. y Díaz Villa, M. (2005). La formación de profesores universitarios para la flexibilidad curricular mediante estrategias virtuales. En: Duart, J. 7 Lupiáñez, F. (coords.). Las TIC en la universidad: estrategia y transformación institucional. Revista de Universidad y Sociedad del Conocimiento (RUSC) [monográfico en línea] [Acceso 16 de abril de 2017]; 2(1). Disponible en: http://www.uoc.edu/rusc/dt/esp/nieto0405.pdf>

Orozco Fuentes, B. (2009). Origen de la noción flexibilidad y su llegada al campo del currículum. En: Orozco Fuentes, B. (coord.) (2009). Currículum: experiencias y configuraciones conceptuales en México. México: IISUE-UNAM / Plaza y Valdés

Orozco Silva, L. E. (2010).Calidad académica y relevancia social de la educación superior en América Latina. En: Revista Iberoamericana de Educación Superior, Instituto de Investigaciones sobre la Universidad y la Educación; 1(1), 24-36. México. Disponible en: https://www. redalyc.org/pdf/2991/299128587002.pdf

Orozco Silva, L. E. (2012). Políticas de Educación Superior y su impacto en las instituciones. Universidad de los Andes Facultad de Administración Bogotá. Disponible en: http:// bdigital.unal.edu.co/7396/1/luisenriqueorozcosilva.20121.pdf

Penalva B., J. (2008). Análisis crítico de los aspectos teóricos del currículum flexible y abierto. Consecuencias educativas. Profesorado. Revista de currículum y formación del profesorado, 11(3), 3-14. Disponible en: http://www.ugr.es/local/recfpro/rev113COL2.pdf

Rodrigo, L. (2006). Organismos Internacionales y Reformas Educativas. El Banco Mundial, la OEI y la política de descentralización educativa en la Argentina de los años noventa. Revista de Educación, 341, 845-866. Disponible en: http://www.revistaeducacion.mec. es/re341_35.html

Roncal, X. (2014). En la búsqueda de la calidad universitaria. Bolivia, Colombia, Cuba y México. Revista Integra Educativa, 7(2), 137-158. Disponible en: http://www.scielo.org.bo/scielo.php? script=sci_arttext\&pid=S1997-40432014000200009\&lng=es\&tlng=es

Sarzuri-Lima, M. (2014). La fascinación educativa por la calidad. Una revisión crítica sobre las tendencias en la conceptualización de la "calidad en la educación". Revista Integra Educativa, 7(2): 71-89. Disponible en: http://www.scielo.org.bo/scielo.php?script=sci_ arttext\&pid=S1997-40432014000200005

Stenhouse, L. (1991). Investigación y desarrollo del Currículum. Madrid: Morata

Tarabini, A. y Bonal, X. (2011). Globalización y política educativa: los mecanismos como método de estudio. Revista de Educación, 235-255. Disponible en: http://www.revistaeducacion. mec.es/re355/re355_10.pdf

Vega Cantor, R. (2014). La calidad educativa una noción neoliberal, propia del darwinismo pedagógico. Revista Integra Educativa, 7(2), 113-125. Disponible en: http://www.scielo.org. bo/scielo.php?script=sci_arttext\&pid=S1997-40432014000200007\&lng=es\&tlng=es 